\title{
Crystalline phases by an improved gradient expansion technique
}

\author{
S. Carignano and M. Mannarelli \\ INFN, Laboratori Nazionali del Gran Sasso, Via G. Acitelli, 22, I-67100 Assergi (AQ), Italy \\ F. Anzuini and O. Benhar \\ INFN and Dipartimento di Fisica, Sapienza Università di Roma, I-00185 Roma, Italy
}

(Received 4 December 2017; published 12 February 2018)

\begin{abstract}
We develop an innovative technique for studying inhomogeneous phases with a spontaneous broken symmetry. The method relies on the knowledge of the exact form of the free energy in the homogeneous phase and on a specific gradient expansion of the order parameter. We apply this method to quark matter at vanishing temperature and large chemical potential, which is expected to be relevant for astrophysical considerations. The method is remarkably reliable and fast as compared to performing the full numerical diagonalization of the quark Hamiltonian in momentum space and is designed to improve the standard Ginzburg-Landau expansion close to the phase transition points. For definiteness, we focus on inhomogeneous chiral symmetry breaking, accurately reproducing known results for one-dimensional and two-dimensional modulations and examining novel crystalline structures, as well. Consistently with previous results, we find that the energetically favored modulation is the so-called one-dimensional realkink crystal. We propose a qualitative description of the pairing mechanism to motivate this result.
\end{abstract}

DOI: 10.1103/PhysRevD.97.036009

\section{INTRODUCTION}

QCD at nonzero baryonic densities is expected to exhibit a rich variety of phases [1]. At sufficiently large values of the quark chemical potential, $\mu$, the chirally broken and confined phase should turn into a chirally restored deconfined phase. This phase transition is accompanied by the melting of the chiral condensate and the possible formation of a color superconducting condensate [2]. These interesting phenomena are expected to occur in a regime where perturbative QCD computations are insufficient and $a b$ initio lattice simulations are currently unavailable due to the sign problem; see, for example, Ref. [3]. Thus, effective models such as the Nambu-Jona-Lasinio (NJL) model are commonly used to describe this region of the phase diagram (see Refs. [4,5] for reviews).

Remarkably, model calculations indicate that various inhomogeneous phases may arise in quark matter at high density. Two notable examples are the crystalline color superconducting phase and the inhomogeneous chiral symmetry broken $(\chi \mathrm{SB})$ phase (see Refs. [6,7] for reviews). The former is probably located at the onset of the deconfined phase, for neutral and beta equilibrated

Published by the American Physical Society under the terms of the Creative Commons Attribution 4.0 International license. Further distribution of this work must maintain attribution to the author(s) and the published article's title, journal citation, and DOI. Funded by SCOAP . matter, while the latter is expected to arise between the homogeneous $\chi \mathrm{SB}$ phase and the chirally restored phase if the quark-antiquark coupling strength is sufficiently large.

More specifically, the inhomogeneous $\chi \mathrm{SB}$ island extends from zero temperature to the chiral critical point, which then turns into a Lifshitz point where three phases (homogeneous $\chi \mathrm{SB}$, inhomogeneous $\chi \mathrm{SB}$, and the chirally restored phase) coexist [8]. The onset of this region, separating it from the traditional homogeneous $\chi \mathrm{SB}$ phase on the left, can be characterized by either a first- or secondorder phase transition, depending on the crystalline shape assumed by the chiral condensate [7]. On the other hand, the chiral restoration transition is always found (at least in the chiral limit) to be a second-order one in which the chiral condensate gradually melts to zero. A remarkable consequence of this is that the position of the chiral restoration transition is independent of the type of crystalline structure considered. Regarding the color superconducting phases, it is known that at asymptotic densities the (spatially homogeneous) color-flavor locked phase [9] is favored. At densities relevant for compact stars, this homogeneous phase could nevertheless be superseded by a crystalline color superconducting phase, especially when the constraints of charge neutrality and beta equilibrium are considered. However, whether this phase transition is of the first or second order is not yet established. The transition to the normal phase should be of the second order, although some modulations seem to indicate a firstorder phase transition. The form of the crystalline pattern 
has only been semiquantitatively established in Ref. [10] by a Ginzburg-Landau (GL) expansion.

Having determined the existence of an inhomogeneous island in the phase diagram, it is natural to ask which crystalline structure will be the most favored one in this region. For definiteness, we focus in this work on the inhomogeneous $\chi \mathrm{SB}$ phase (but we will comment on applications to the crystalline color superconducting phase, as well). Contrary to the case of crystalline colorsuperconductivity, mean-field model calculations on the inhomogeneous $\chi \mathrm{SB}$ phase seem to indicate that the favored type of modulation is a one-dimensional real structure called a "real-kink crystal," which can be expressed in terms of Jacobi elliptic functions $[7,11,12]$. In particular, twodimensional structures are found to be strongly disfavored compared to the one-dimensional ones, both in the vicinity of the Lifshitz point [13] as well as at zero temperature [14]. The latter result has been obtained through a computationally expensive analysis. Indeed, even within the NJL model in the mean-field approximation, the evaluation of the free energy for inhomogeneous phases is a nontrivial task. One way to obtain it is by performing a full diagonalization of the quark Hamiltonian and sum over its eigenvalues; alternatively, GL expansions including gradient terms of the order parameter can be used. Both methods, however, have their own limitations: the diagonalization of the Hamiltonian can be performed analytically only in very special cases, while for most types of modulations, one has to resort to a bruteforce numerical computation in momentum space $[14,15]$. On the other hand, the GL expansion of the free energy in the order parameter and its gradients is expected to be valid only close to the second-order transition to the chirally restored phase $[10,13,16]$. But gradients of the order parameter may not vanish sufficiently fast, even close to the second-order phase transition point, making the GL expansion power counting nontrivial. One notable exception is the Lifshitz point, where both the order parameter and its gradient are expected to vanish. At any rate, while the determination of the GL coefficients is in principle straightforward, in the presence of an inhomogeneous order parameter, the actual derivation of the GL energy functional becomes extremely tedious at higher orders. The number of possible terms steadily increases, and no automated procedure for the derivation of the coefficients has yet been developed. So far, for inhomogeneous chiral condensates expansions up to the order 8 have been derived [13].

In this work, with the aim of going beyond these limitations, we build a controlled framework for investigating inhomogeneous phases away from the Lifshitz point (for the astrophysical relevant case of matter at vanishing temperature) without having to resort to a brute-force numerical diagonalization of the quark Hamiltonian in momentum space. For this, we devise an improved Ginzburg-Landau (IGL) approximation which can correctly describe both phase transitions delimiting the inhomogeneous phase to (at least in principle) arbitrary precision. This is done on one hand by implementing "by construction" a correct description of the homogeneous phase which also contains information on long-wavelength modulations of the chiral condensate and on the other hand by incorporating a sufficiently large number of appropriate gradient terms. The latter can be determined straightforwardly without having to resort to the full computation of the GL coefficients thanks to our analytical knowledge of the eigenvalue spectrum of a simple modulation of the condensate, namely, a single plane wave.

This paper is organized as follows. In Sec. II, we introduce the IGL approximation to describe the inhomogeneous phases, focusing on the $\chi \mathrm{SB}$ case. In Sec. III, we extract the coefficients of the IGL expansion governing the transition to the chirally restored phase starting from the single plane wave modulation. In Sec. IV, we compare the results of the GL and IGL approximation with the numerical results of the diagonalization of the full quark Hamiltonian. In Sec. V, we analyze two-dimensional (2D) modulations including a novel ansatz. A qualitative discussion of the pairing mechanism is given in Sec. VI. We finally draw our conclusions in Sec. VII.

\section{IMPROVED GINZBURG-LANDAU EXPANSION}

To develop our formalism, we focus on the phenomenon of inhomogeneous $\chi \mathrm{SB}$ breaking starting from a GL expansion for the free energy in the NJL model within the mean-field approximation [4,5,17].

The idea behind the GL expansion is that close to the Lifshitz point the thermodynamic potential can be written as an expansion in powers of the chiral order parameter $M(z)=-2 G[S(z)+i P(z)]$ and its spatial derivatives (here, $S(z)=\langle\bar{\psi} \psi\rangle$ and $P(z)=\left\langle\bar{\psi} i \gamma^{5} \tau^{3} \psi\right\rangle$ are the scalar and pseudoscalar chiral condensates, respectively, and $G$ is the scalar coupling constant in the NJL Lagrangian). More specifically, for a real modulation, one can write $[8,13]$

$$
\begin{aligned}
\Omega_{\mathrm{GL}}= & \Omega[0]+\frac{1}{V} \int d \mathbf{x}\left[\alpha_{2} M^{2}+\alpha_{4}\left(M^{4}+(\nabla M)^{2}\right)\right. \\
& +\alpha_{6}\left(M^{6}+3(\nabla M)^{2} M^{2}+\frac{1}{2}\left(\nabla M^{2}\right)^{2}+\frac{1}{2}\left(\nabla^{2} M\right)^{2}\right) \\
& +\alpha_{8}\left(M^{8}+14 M^{4}(\nabla M)^{2}-\frac{1}{5}(\nabla M)^{4}\right. \\
& +\frac{18}{5} M\left(\nabla^{2} M\right)(\nabla M)^{2} \\
& \left.\left.+\frac{14}{5} M^{2}\left(\nabla^{2} M\right)^{2}+\frac{1}{5}\left(\nabla^{3} M\right)^{2}\right)+\cdots\right]
\end{aligned}
$$

where $\alpha_{n}$ are some coefficients depending on the microscopic model. The reasoning behind this expansion is that terms with the same $\alpha_{n}$ are equally important. In other words, close to the Lifshitz point, both $M$ and $\nabla M$ are small; thus, $M^{n}$ and $\nabla^{m} M^{n-m}$, with $n>m$, can be comparable. 
However, this is a very special case because approaching the second-order phase transition $M$ is expected to vanish, but $(\nabla M) / M$ may be nonzero. In particular, at $T=0$, and close to the phase transition to the chirally restored phase, the power counting underlying Eq. (1) is expected to be incorrect [7]; more specifically, $\nabla^{m} M^{n-m}$ terms can be larger than the $M^{n}$ terms. In principle, we expect this to happen for any sufficiently small temperature away from the Lifshitz point, but in the following, we will focus for simplicity on the $T=0$ case, which is relevant for sufficiently old compact stars. Furthermore, Eq. (1) is insufficient to provide a reasonable description of the homogeneous $\chi \mathrm{SB}$ phase. This calls for a different scheme and/or a different approach.

From a technical point of view, the GL coefficients $\alpha_{n}$ in the NJL model can be easily determined, either from their general expression [8] or, more pragmatically, by evaluating the free energy for a homogeneous order parameter and performing an expansion in powers of $M$, isolating the coefficients multiplying the $M^{n}$ terms. The knowledge of the $\alpha_{n}$ is, however, insufficient to build a GL functional for inhomogeneous condensates, as different gradient terms of the same order carry different relative prefactors compared to the $M^{n}$ term [see Eq. (1)]. The evaluation of these terms in the inhomogeneous $\chi \mathrm{SB}$ phase is already quite tedious at order $\alpha_{6}$ and becomes increasingly more involved at higher orders [13].

To improve the GL scheme and to circumvent the above technical difficulties, let us take one step back and inspect the structure of Eq. (1). There, we have ordered the terms in such a way that order by order the first one is $M^{n}$ and the last one is $\left(\nabla^{\frac{n}{2}-1} M\right)^{2}$. As already noted, these two sets of terms are particularly relevant; indeed, they are the dominant contributions close to the phase transitions. In particular, the $\left(\nabla^{\frac{n}{2}-1} M\right)^{2}$ terms are the dominant gradient contributions close to the chirally restored phase because terms with a higher power of $M$ are suppressed. The $M^{n}$ terms, on the other hand, are particularly relevant close to the transition to the homogeneous $\chi \mathrm{SB}$ phase (indeed, these are the only terms present in the homogeneous phase, in which gradients vanish). However, the free energy of the homogeneous $\chi \mathrm{SB}$ phase is known in an analytical form. These considerations motivate the following IGL expansion, which for a real order parameter reads

$$
\begin{aligned}
\Omega_{\mathrm{IGL}}= & \frac{1}{V} \int d \mathbf{x}\left[\Omega_{\mathrm{hom}}\left(\overline{M^{2}}\right)+\alpha_{6}\left(3(\nabla M)^{2} M^{2}+\frac{1}{2}\left(\nabla M^{2}\right)^{2}\right)\right. \\
& +\alpha_{8}\left(14 M^{4}(\nabla M)^{2}-\frac{1}{5}(\nabla M)^{4}+\frac{18}{5} M\left(\nabla^{2} M\right)(\nabla M)^{2}\right. \\
& \left.\left.+\frac{14}{5} M^{2}\left(\nabla^{2} M\right)^{2}\right)+\sum_{n \geq 1} \tilde{\alpha}_{2 n+2}\left(\nabla^{n} M\right)^{2}\right]
\end{aligned}
$$

where the first and the last terms in the square bracket characterize this novel expansion technique.
In particular, $\Omega_{\text {hom }}\left(\overline{M(z)^{2}}\right)$ is the free energy for a homogeneous order parameter, evaluated point by point for a moving average of the mass function defined as

$$
\overline{M(z)^{2}}=\frac{1}{\lambda} \int_{z-\lambda / 2}^{z+\lambda / 2} M^{2}(\xi) d \xi
$$

where, as we will see, the relevant wavelength scale $\lambda$ is determined by the chemical potential $\mu$. If $M$ is spatially constant, this term gives by construction the free energy of the homogeneous $\chi \mathrm{SB}$ phase. On the other hand, for a general oscillation, it captures the long-wavelength modulation of the condensate; from the point of view of an effective field theory, this can be seen as the dominant term for long-wavelength fluctuations, while high-frequency components have been integrated out. The $\left(\nabla^{n} M\right)^{2}$ term is instead the dominant one at high frequencies, of the same order as or higher than $\mu$. Indeed, the last term in Eq. (2) includes the leading gradient contributions close to the second-order transition to the chirally restored phase. The requirement for this term to be dominant is the vanishing of the amplitude of the chiral condensate, which is what happens close to the second-order transition to the normal phase. We labeled the coefficients multiplying these gradient terms as $\tilde{\alpha}_{n}$, as they will be equal to the $\alpha_{n}$ up to a numerical prefactor. By inspecting Eq. (1), we can see immediately that $\tilde{\alpha}_{4}=\alpha_{4}, \quad \tilde{\alpha}_{6}=\alpha_{6} / 2$, and $\tilde{\alpha}_{8}=\alpha_{8} / 5$, whereas for higher orders, these relations have not been determined until now.

The first and the last terms in the expansion in Eq. (2) thus guarantee the agreement with the exact result close to the two phase transitions. The other terms, which are taken from the traditional GL expansion, are expected to be relevant in the region between the two phase transitions, when $\nabla M \sim M^{2}$, or more precisely when the modulation wavelength, $\lambda$, satisfies $|M| \sim 1 / \lambda$. As we will see in the following, the $\alpha_{6}$ terms will be sufficient to provide a good qualitative agreement with the full numerical results, and including the $\alpha_{8}$ terms will allow us to obtain an excellent quantitative agreement throughout the whole inhomogeneous $\chi \mathrm{SB}$ phase.

The IGL prescription can, of course, be generalized to complex modulations: for our novel terms, this amounts to simply replacing $M^{2}$ by $|M|^{2}$ in the moving average Eq. (3) and $\left(\nabla^{n} M\right)^{2}$ by $\left|\nabla^{n} M\right|^{2}$ in the leading gradient terms.

One important aspect is that, when derived from an NJL model, the $\alpha_{n}$ coefficients do not only depend on $\mu$ but are also sensitive to the regularization scale, $\Lambda$. However, once this scale is fixed, the coefficients themselves do not depend on the considered shape of the modulation of the condensate. At vanishing temperature and for a PauliVillars regularization with three counterterms, a regulator $\Lambda$, and coefficients $c_{0}=1, c_{1}=-3, c_{2}=3, c_{3}=-1$ (see Ref. [12]), we find 


$$
\begin{aligned}
& \alpha_{2}=\frac{1}{4 G}-\frac{N_{f} N_{c}}{8 \pi^{2}}\left(3 \Lambda^{2} \log \left(\frac{4}{3}\right)-2 \mu^{2}\right), \\
& \alpha_{4}=-\frac{N_{f} N_{c}}{16 \pi^{2}} \log \left(\frac{32 \mu^{2}}{3 \Lambda^{2}}\right), \\
& \alpha_{6}=\frac{N_{f} N_{c}}{96 \pi^{2}}\left(\frac{11}{3 \Lambda^{2}}+\frac{1}{\mu^{2}}\right), \\
& \alpha_{8}=\frac{N_{f} N_{c}}{256 \pi^{2}}\left(\frac{1}{2 \mu^{4}}-\frac{85}{27 \Lambda^{4}}\right),
\end{aligned}
$$

where $N_{f}$ and $N_{c}$ are the numbers of quark flavors and colors, respectively.

\section{HIGHER-ORDER GRADIENTS FROM A CDW MODULATION}

The only missing ingredients in the IGL expansion of Eq. (2) are the $\tilde{\alpha}_{n}$ coefficients for $n>8$. We compute them by exploiting the analytical knowledge of the eigenvalue spectrum of the quark Hamiltonian for the so-called chiral density wave (CDW) ansatz

$$
M(z)=\Delta e^{2 i q z},
$$

corresponding to a static single plane wave modulation, chosen without loss of generality along the $z$ direction, with amplitude $\Delta$ and wave number $2 q$. For this simple case, the quasiparticle dispersion law is known $[18,19]$,

$$
E_{\epsilon}=\sqrt{p_{\perp}^{2}+\left|E_{z}+\epsilon q\right|^{2}},
$$

where $\epsilon= \pm 1, p_{\perp}=\sqrt{p_{x}^{2}+p_{y}^{2}}$, and $E_{z}=\sqrt{p_{z}^{2}+\Delta^{2}}$. At vanishing temperature, the free energy for this modulation is given by

$$
\begin{aligned}
\Omega_{\mathrm{CDW}}= & -\frac{N_{f} N_{c}}{4 \pi^{2}} \int_{0}^{\infty} d p_{\perp} p_{\perp} \\
& \times \int_{-\infty}^{\infty} d p_{z} \sum_{\epsilon= \pm}\left[E_{P V}^{\epsilon}+\left(\mu-E^{\epsilon}\right) \theta\left(\mu-E^{\epsilon}\right)\right]+\frac{\Delta^{2}}{4 G},
\end{aligned}
$$

where again the Pauli-Villars regularization scheme has been adopted; see Refs. $[4,7,12]$. We now expand the free energy in a Taylor-like series,

$$
\begin{aligned}
\Omega & =\Omega_{0}+\left.\frac{\partial \Omega}{\partial\left(\Delta^{2}\right)}\right|_{\Delta=0} \Delta^{2}+\left.\frac{1}{2} \frac{\partial^{2} \Omega}{\partial\left(\Delta^{2}\right)^{2}}\right|_{\Delta=0} \Delta^{4}+\cdots \\
& =\Omega_{0}+\Omega_{2} \Delta^{2}+\Omega_{4} \Delta^{4}+\cdots
\end{aligned}
$$

starting from $\Omega_{0}=\Omega(\Delta=0)$. Each term can be separated in a vacuum contribution $\Omega_{n}^{V}$ and a medium contribution $\Omega_{n}^{\mu}$ which (at $T=0$ ) depends on the quark chemical potential $\mu$. For example, the zeroth order is

$$
\Omega_{0}^{\mu}=-\frac{N_{f} N_{c}}{4 \pi^{2}} \mu^{4},
$$

which is minus the pressure of a free Fermi gas of massless particles and is $q$ independent, as it should be.

The first nontrivial term is proportional to $\Delta^{2}$ and is given by

$$
\begin{aligned}
\Omega_{2}= & \frac{1}{4 G}-\frac{N_{f} N_{c}}{4 \pi^{2}} \lim _{\Delta \rightarrow 0} \int_{0}^{\infty} d p_{\perp} p_{\perp} \\
& \times \int_{-\infty}^{\infty} d p_{z} \sum_{\epsilon= \pm} \frac{1}{2 E_{\epsilon}}\left(1+\epsilon \frac{q}{\sqrt{p_{z}^{2}+\Delta^{2}}}\right) \\
& \times\left[\sum_{j} c_{j} \frac{E_{\epsilon}}{\sqrt{E_{\epsilon}^{2}+j \Lambda^{2}}}-\theta\left(\mu-E_{\epsilon}\right)\right] \\
= & \Omega_{2}^{\text {cond }}+\Omega_{2}^{V}(0)+\Omega_{2}^{V}(q)+\Omega_{2}^{\mu}(0)+\Omega_{2}^{\mu}(q),
\end{aligned}
$$

where the first term is simply a constant due to the condensation energy, and we write down explicitly the Pauli-Villars regularization of the vacuum part. Furthermore, we isolated the medium and vacuum $q$ dependent contributions to $\Omega_{2}$, which can be evaluated analytically,

$$
\begin{aligned}
\Omega_{2}^{V}(q)= & -\frac{N_{f} N_{c}}{4 \pi^{2}} q \sum_{j=0}^{3} c_{j}\left[\left(q-\sqrt{q^{2}+j \Lambda^{2}}\right) \log \left(j \Lambda^{2}\right)\right. \\
& \left.+2 \sqrt{q^{2}+j \Lambda^{2}} \log \left(q+\sqrt{q^{2}+j \Lambda^{2}}\right)\right]
\end{aligned}
$$

and

$$
\begin{aligned}
\Omega_{2}^{\mu}(q)= & \frac{N_{f} N_{c}}{4 \pi^{2}} q\left[(\mu-q) \log \left(\frac{|\mu-q|}{q}\right)\right. \\
& \left.-(\mu+q) \log \left(\frac{\mu+q}{q}\right)\right] .
\end{aligned}
$$

Upon closer inspection, we note that both contributions carry a $\log (q)$ term which could possibly spoil any expansion. However, by adding them up, these log pieces cancel out. Expanding in $q / \mu$, we obtain

$$
\begin{aligned}
\Omega_{2}(q)= & \frac{N_{f} N_{c}}{4 \pi^{2}} \mu^{2}\left[-\log \left(\frac{32 \mu^{2}}{3 \Lambda^{2}}\right)\left(\frac{q}{\mu}\right)^{2}+\left(\frac{1}{3}+\frac{11 \mu^{2}}{9 \Lambda^{2}}\right)\left(\frac{q}{\mu}\right)^{4}\right. \\
& \left.+\left(\frac{1}{10}-\frac{17 \mu^{4}}{27 \Lambda^{4}}\right)\left(\frac{q}{\mu}\right)^{6}+\left(\frac{1}{21}+\frac{230 \mu^{6}}{567 \Lambda^{6}}\right)\left(\frac{q}{\mu}\right)^{8}+\cdots\right],
\end{aligned}
$$

which is a controlled expansion as long as $q<\mu$. This typically turns out to be the case, as we will see in the following sections. Furthermore, from this expansion, we 
can obtain all the $\tilde{\alpha}_{n}$ terms required for the IGL expansion. Indeed, by inspecting Eq. (2), it is clear that for the CDW all the terms in the form $\left|\nabla^{\frac{n}{2}} M\right|^{2}$ turn into $q^{n} \Delta^{2}$ terms and are therefore all contained in $\Omega_{2}$. Thus, by expanding $\Omega_{2}$ in powers of $q$ as in Eq. (13), we can extract these terms to arbitrarily high order in an extremely simple way. Comparing the lower-order coefficients with the expressions in Eq. (4), we see that they agree, and pushing our expansion to higher orders, we find, for example,

$$
\tilde{\alpha}_{10}=\frac{N_{f} N_{c}}{1024 \pi^{2}}\left(\frac{230}{567 \Lambda^{6}}+\frac{1}{21 \mu^{6}}\right) \text {. }
$$

From the above expansion, it is clear that the relevant frequencies are of the order of $\mu$, suggesting that the scale to be employed in the moving average Eq. (3) should be of the order of $1 / \mu$. This scale is also comparable to the radius for the single soliton introduced in Ref. [20] for the real-kink crystal modulation (see later), $R_{\mathrm{sol}}=\pi /\left(\sqrt{12} M_{\mathrm{vac}}\right)\left(M_{\mathrm{vac}}\right.$ being the vacuum constituent quark mass), since the inhomogeneous phase is typically realized for $\mu \sim M_{\mathrm{vac}}$. In the following, we will choose $\lambda=1 / \mu$, although any other choice in the same ballpark leads to similar results.

\section{BENCHMARKS AND APPLICATIONS OF THE IGL}

We are now ready to evaluate and minimize the IGL free energy and compare it with the standard GL approximation [including up to $\mathcal{O}\left(\alpha_{8}\right)$ terms; see Eq. (1)] and the full numerical result. We begin by considering two different one-dimensional (1D) modulations of the condensate, in order to explore the reliability of the GL and IGL expansions. We work in the chiral limit using a PauliVillars regulator $\Lambda=757.048 \mathrm{MeV}$ and a scalar coupling $G=6.002 / \Lambda^{2}$, corresponding to a vacuum constituent quark mass $M_{\text {vac }}=300 \mathrm{MeV}$ and a pion decay constant $f_{\pi}=88 \mathrm{MeV}$ [12]. We remind that all of our results are obtained at zero temperature.

\section{A. Chiral density wave}

We start by considering the CDW modulation; see Eq. (5). In Fig. 1, we report the results obtained for the variational parameters $\Delta, q$ (top panel) and the free energy at the minimum (bottom panel). For the single plane wave, the numerical results (solid black line) are extremely reliable and can be used as a benchmark to test the GL (dashed blue line) and the IGL (dotted red line) approximations. As a first step, we truncated the IGL approximation to order $\tilde{\alpha}_{10}$. The three approaches give qualitatively similar results, showing that in this case both $\Delta$ and $q$ are discontinuous at the phase transition between the homogeneous and inhomogeneous $\chi \mathrm{SB}$ phases. The value of $q$ vanishes in the homogeneous $\chi \mathrm{SB}$ phase and jumps to about $200 \mathrm{MeV}$ at the onset of the inhomogeneous $\chi \mathrm{SB}$
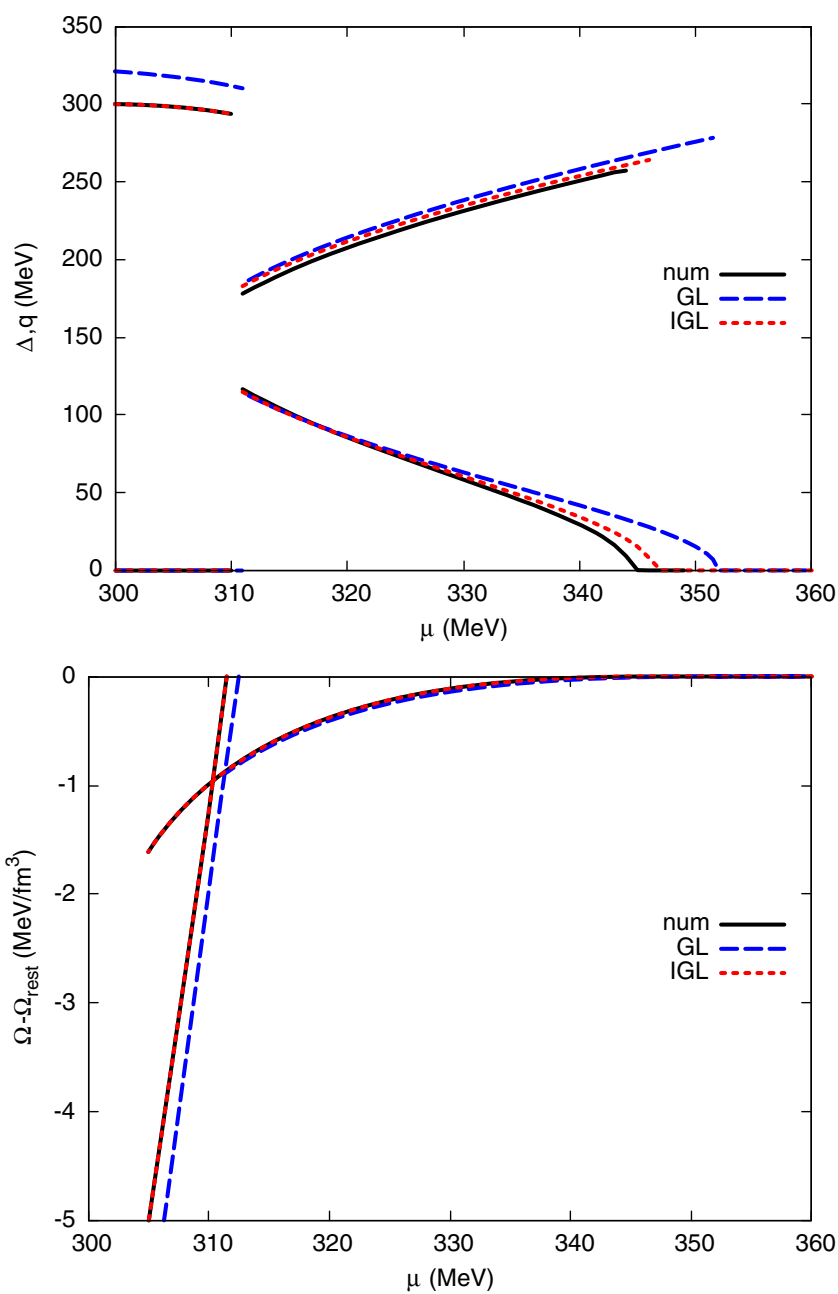

FIG. 1. Comparison of the numerical results with the GL expansion and the IGL approximation for the CDW modulation as a function of the quark chemical potential. Top: values of $\Delta$ (curves with a decreasing behavior) and $q$ (curves with an increasing behavior) that minimize the free energy. Both GL and IGL give good results in the inhomogeneous phase. The GL tends to favor more the inhomogeneous phase over the chirally restored phase, and in the homogenous $\chi \mathrm{SB}$ phase, the GL expansion tends to overestimate $\Delta$. Bottom: free energy (after subtraction of the free energy of the chirally restored phase, $\Omega_{\text {rest }}$ ). The approximate expressions almost overlap with the numerical ones in the inhomogeneous $\chi \mathrm{SB}$ phase. However, in the homogenous $\chi$ SB phase, only the IGL approximation leads to a good agreement with the numerical results.

phase. Then, it monotonically increases, as a function of $\mu$, in the inhomogeneous phase. The $\Delta$ parameter is instead about $M_{\mathrm{vac}}=300 \mathrm{MeV}$ in the homogenous $\chi \mathrm{SB}$ phase and then a decreasing function of $\mu$ in the inhomogeneous phase, eventually vanishing at the second-order transition to the chirally restored phase.

The first remarkable result visible by inspecting Fig. 1 is that, even at zero temperature, the standard GL expansion provides a good quantitative agreement with the results of the full numerical computation. It fails, however, to 
properly reproduce the numerical results in two key regions: close to the transition to the chirally restored phase, where it overshoots the transition point, and at the transition between the homogeneous and inhomogeneous $\chi \mathrm{SB}$ phases, failing to correctly reproduce the value of $\Delta$ in the homogeneous $\chi \mathrm{SB}$ phase and the transition point. On the other hand, the IGL exactly does what it is designed for: it improves the description of these two regions. Most notably, it exactly reproduces the free energy in the homogeneous phase and the transition to the inhomogeneous $\chi \mathrm{SB}$ phase. Moreover, it shifts the transition to the chirally restored phase closer to the numerical result.

It is important to stress that close to the chiral restoration transition the IGL is designed for giving a systematic controlled improvement over the standard GL by the inclusion of higher-order $\left|\nabla^{n} M\right|^{2}$ terms, which, as already discussed, can be straightforwardly extracted from Eq. (13). This is shown in Fig. 2, where we can see how the secondorder transition is increasingly better described as we include higher-order terms. In particular, we can see that in order to get a good qualitative agreement we need at least the $\mathcal{O}\left(\alpha_{8}\right)$ terms; otherwise, the inhomogeneous $\chi$ SB phase extends to arbitrarily high chemical potentials. We can interpret this result by inspecting Eq. (13), or equivalently the expressions for the GL coefficients: close to the chiral restoration transition and for reasonable values of $\mu / \Lambda$, the leading $\mathcal{O}\left(\alpha_{4}\right)$ coefficient is negative and provides an energy gain in the formation of an inhomogeneous phase, whereas higher-order terms constitute energy costs. Indeed, while we

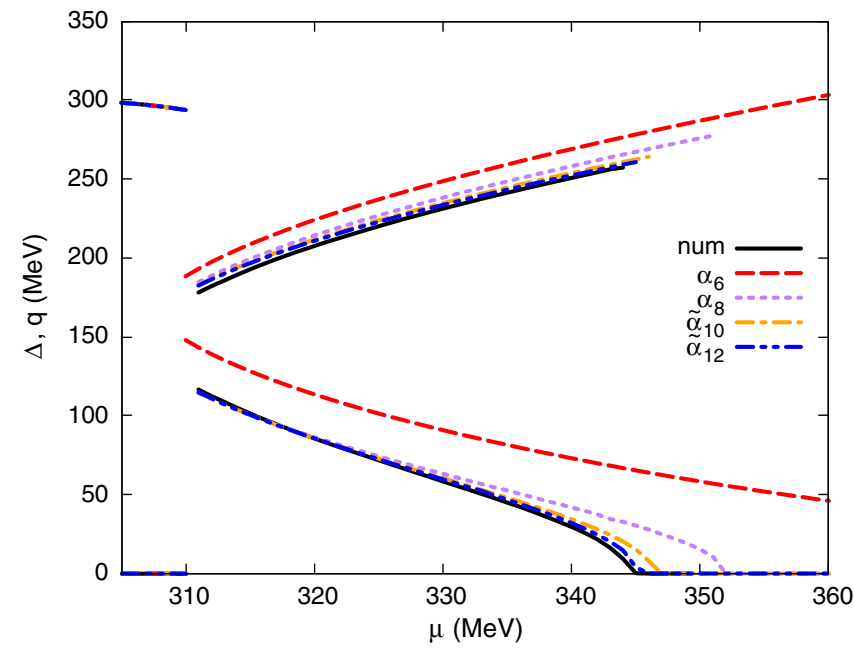

FIG. 2. Analysis of the IGL approximation for the CDW condensate. The various lines correspond to the values of $\Delta$ (curves with a decreasing behavior) and $q$ (curves with an increasing behavior) that minimize the free energy, as a function of the quark chemical potential. Solid lines are results obtained by the full diagonalization of the quark Hamiltonian, and the others correspond to the IGL expansion, Eq. (2), including gradient terms of different orders. Increasing the number of the gradient terms, the position of the second-order phase transition is increasingly well determined. find that, in principle, within our regularization scheme the coefficients $\alpha_{4 n}$ for $n>1$ might flip sign [see Eq. (4)] and actually favor large $q$ solutions; in practice, this would require chemical potentials too close to the regulator $\Lambda$ for us to trust the model results in that regime. ${ }^{1}$ Therefore, we find that higher-order gradient terms provide (increasingly smaller) energy costs, which gradually push the phase transition toward lower chemical potentials, gradually approaching the full numerical result. In practice, the convergence of this sum turns out to be very rapid: by including $\tilde{\alpha}_{10}$ corrections, we are off the full numerical result for the transition chemical potential by only $2 \mathrm{MeV}$, and the IGL results from order $\tilde{\alpha}_{12}$ on become practically indistinguishable from the numerical result. In light of this, in the following, we will consider for simplicity the truncated IGL expansion at order $\tilde{\alpha}_{10}$, with the understanding that more refined results can be straightforwardly obtained by simply adding higher-order gradient terms.

\section{B. Real-kink crystal}

The results with the CDW ansatz suggest that the IGL approximation works extremely well. For a second check, we compare the GL and IGL results with the numerical ones for the modulation which has been found to be the most favored in the inhomogeneous $\chi \mathrm{SB}$ window, namely, the real-kink crystal (RKC) [7,12]

$$
M(z)=\Delta \sqrt{\nu} \operatorname{sn}(\Delta z \mid \nu),
$$

where $\operatorname{sn}(\Delta z \mid \nu)$ is a Jacobi elliptic function, the shape of which is characterized by $\Delta$ and by the so-called elliptic modulus $\nu$.

After computing the cell averages over $M(z)$ and plugging them in the GL and IGL expression, we obtain the results shown in Fig. 3. Again, we find a good agreement of the GL result with the full computation, while the IGL provides a significant quantitative improvement, reproducing the full numerical results within a few percent error. In this case, the effect of the first term in the IGL expansion in Eq. (2) is more evident. It forces the average value of the condensate to match the homogeneous value, sensibly improving the agreement with the numerical results. This effect is due to the fact that the RKC ansatz can be seen as a superposition on many different waves with different amplitudes. The long-wavelength amplitudes dominate close to the phase transition with the homogeneous $\chi \mathrm{SB}$ phase. On the other hand, for a CDW, there is one single spatial frequency $q$, which is large. Therefore, in that case, the IGL does not improve much with respect to the GL approximation close to this phase transition.

\footnotetext{
${ }^{1}$ This behavior might also be related to the appearance of a second inhomogeneous phase at high chemical potentials within NJL model calculations; see the discussions in Refs. [21,22].
} 

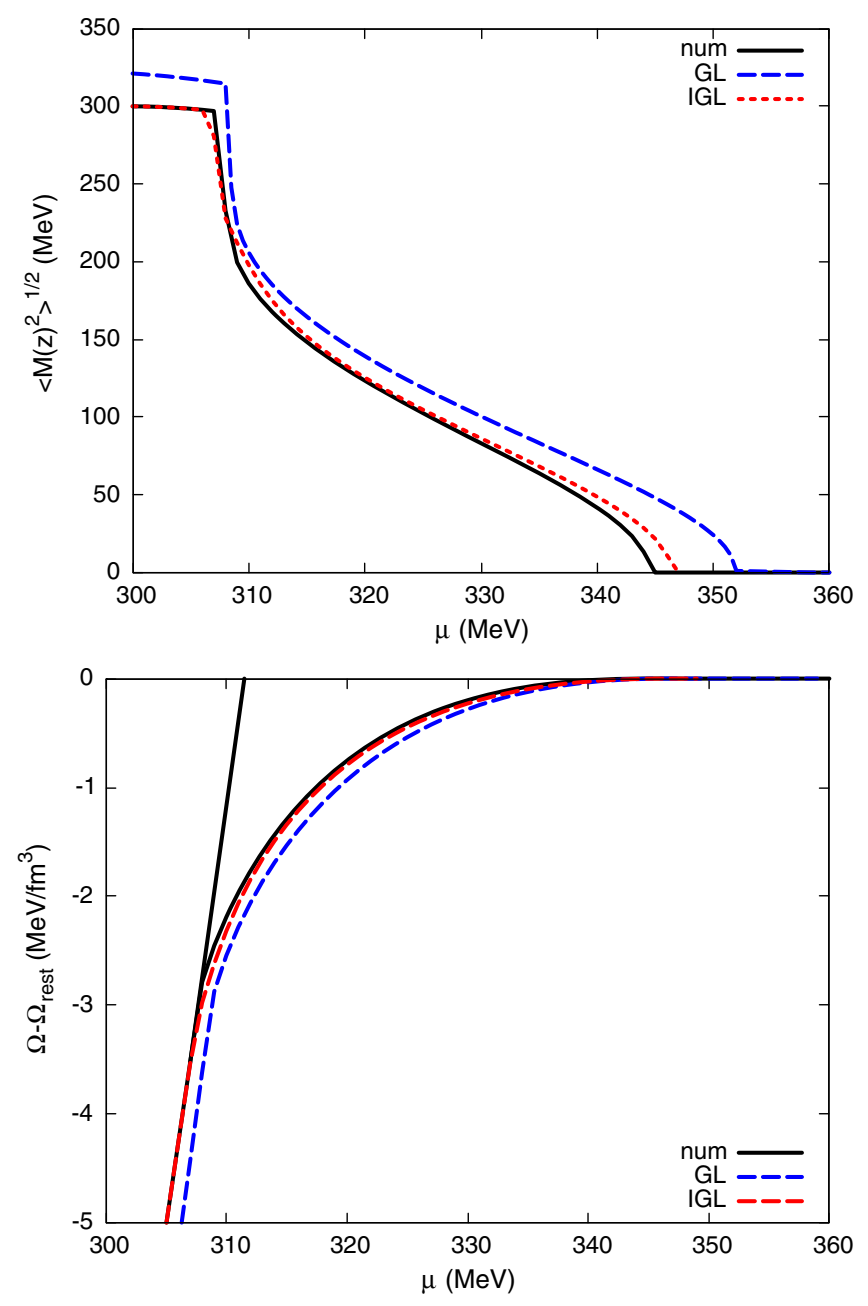

FIG. 3. Comparison of the numerical results with the GL expansion and the IGL approximation for the RKC, Eq. (15). Top: average value of the condensate, $\sqrt{\left\langle M(z)^{2}\right\rangle}$. Bottom: difference between the free energy at the minimum and the free energy of the chirally restored phase.

It might be interesting at this point to compare the spatially modulated quark number density of the system obtained within the GL and IGL approximations with the numerical results of Ref. [23]. In our case, it is simply obtained by differentiating the integrand of Eq. (2) with respect to $\mu$. This basically amounts to an improvement over a "local Fermi-gas" approximation [amounting to simply considering the first term in Eq. (2)], which has already been found to reproduce very well the behavior of the density of the system [24]. Our results are shown in Fig. 4. There, we can see that once again the IGL provides better agreement with the full result as compared to the GL, although in this case, the results do not match perfectly, especially close to the phase transition to the homogeneous broken phase. This is due to the fact that $\Delta$ and $\nu$ give the amplitude and frequency of the density oscillations. Since both are slightly different from the ones obtained by the full numerical calculation, the resulting density has an
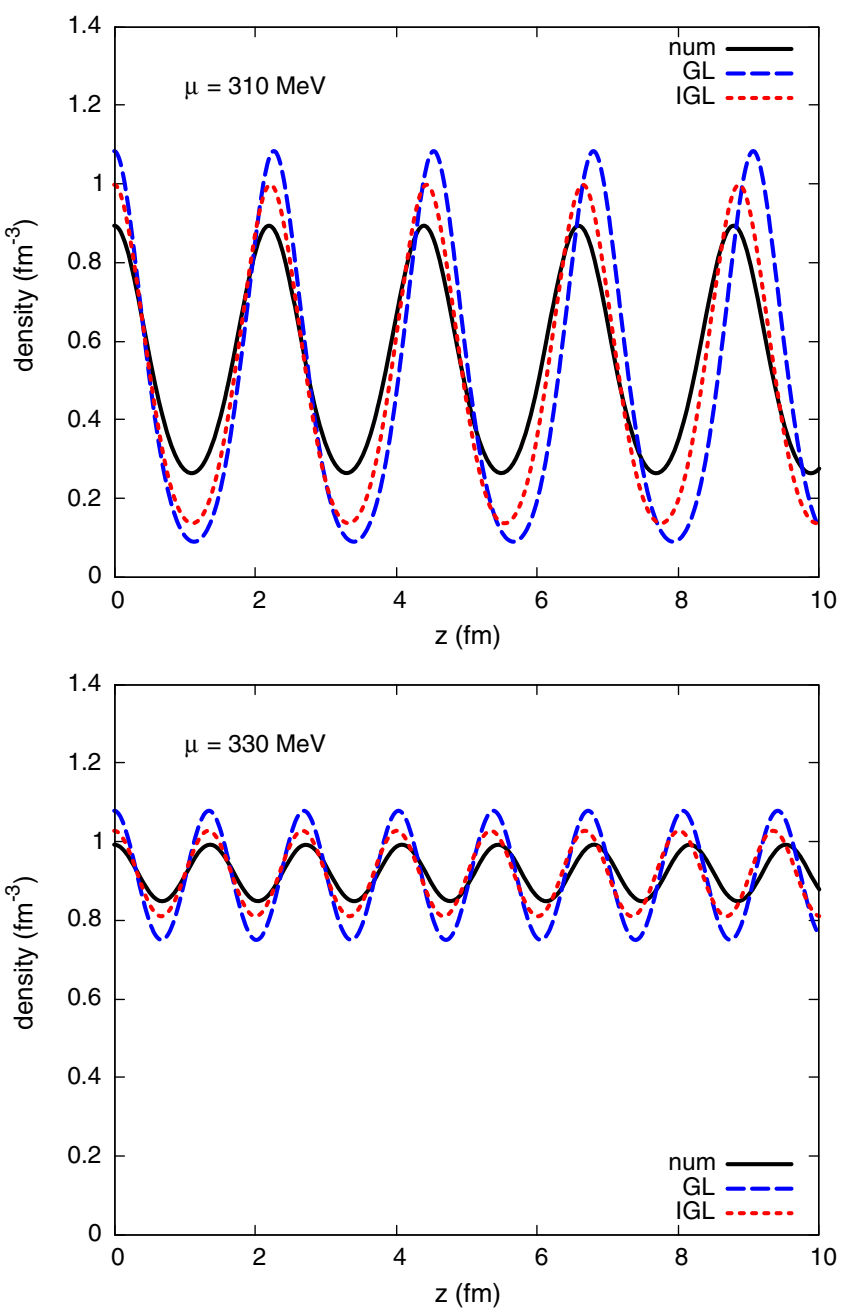

FIG. 4. Comparison of the quark number density obtained by the GL, IGL, and numerical methods for the RKC modulation (15). The IGL always performs better than the GL, with a discrepancy with the numerical method that is larger close to the $\chi \mathrm{SB}$ phase than at the chirally restored phase. The top panel corresponds to $\mu=310 \mathrm{MeV}$, while the bottom panel is obtained for $\mu=330 \mathrm{MeV}$.

amplitude and oscillation period different from the numerical ones.

The RKC modulation is somehow similar to the lasagna phase in nuclear matter, that is a type of pasta phase [25] expected to be realized in the inner crust of compact stars. In this phase, nuclei form sheets immersed in a liquid of nuclear matter. However, with changing densities, the Lasagna phase is supposed to be superseded by different modulations, possibly giving rise to higher-dimensional structures. Following this analogy, one might expect that higher-dimensional modulations can become favored at different values of the quark chemical potential. Another argument in favor of higher-dimensional modulations comes from quarkyonic matter studies, in which it is expected that increasingly complex crystalline structures can be formed by the chiral condensate as the density increases [26]. 


\section{TWO-DIMENSIONAL STRUCTURES}

Since the IGL provides very accurate results for the order parameters and free energies of one-dimensional modulations with minimal computational effort, let us now move on and consider two-dimensional structures. Close to the Lifshitz point, a systematic GL analysis of different types of higher-dimensional modulations has been performed in Ref. [13], while a complementary numerical analysis for the astrophysically relevant $T=0$ case can be found in Ref. [14]. Comparing the IGL results with the numerical ones of Ref. [14] for a two-dimensional square lattice with a sinusoidal ansatz, that is,

$$
M(x, y)=\Delta \cos (q x) \cos (q y),
$$

we obtain the order parameters and the free energies reported in Fig. 5. It is clear that the agreement is again extremely good, ${ }^{2}$ and we recall that the IGL result can be computed with very limited numerical effort [basically amounting to the evaluation of $\left\langle\Omega_{\text {hom }}\left(\overline{M^{2}}\right)\right\rangle$, as all the other terms can be computed analytically].

Using the IGL method, we are in a position to easily test different 2D modulations. First, we consider a square lattice with two RKC-type modulations along the $x$ and $y$ directions, that is,

$$
M(x, y)=\Delta \nu \operatorname{sn}(\Delta x, \nu) \operatorname{sn}(\Delta y, \nu) .
$$

The practical implementation of this modulation in the numerical framework of Ref. [14] would be extremely complicated, as it would in principle require an expansion of the order parameter in a large number of Fourier harmonics and a minimization of the free energy with respect to all of their amplitudes. Instead, within the IGL approximation, it can be straightforwardly implemented in the same way as with the 2D cosine. The minimization of the IGL free energy with respect to $\Delta$ and $\nu$ yields qualitatively similar results to the one-dimensional RKC for the order parameters. When computing the free energy associated with this modulation, we find, similarly to what happens with the one-dimensional modulations, that the RKC-type solution is almost degenerate with the cosine one with the exception of the region close to the onset of the inhomogeneous phase, as shown in Fig. 6. In that figure, we also see that this type of modulation is also disfavored with respect to its one-dimensional counterpart. We performed a further check in this direction by considering the ansatz

$$
M(x, y)=\Delta\left[\sqrt{\nu_{x}} \operatorname{sn}\left(\Delta x, \nu_{x}\right)+\sqrt{\nu_{y}} \operatorname{sn}\left(\Delta y, \nu_{y}\right)\right],
$$

\footnotetext{
${ }^{2}$ It is worth recalling that the numerical results for the $2 \mathrm{D}$ modulations obtained in Ref. [14] may carry some numerical uncertainty due to the cutoffs implemented in the numerical diagonalization of the quark Hamiltonian in momentum space.
}
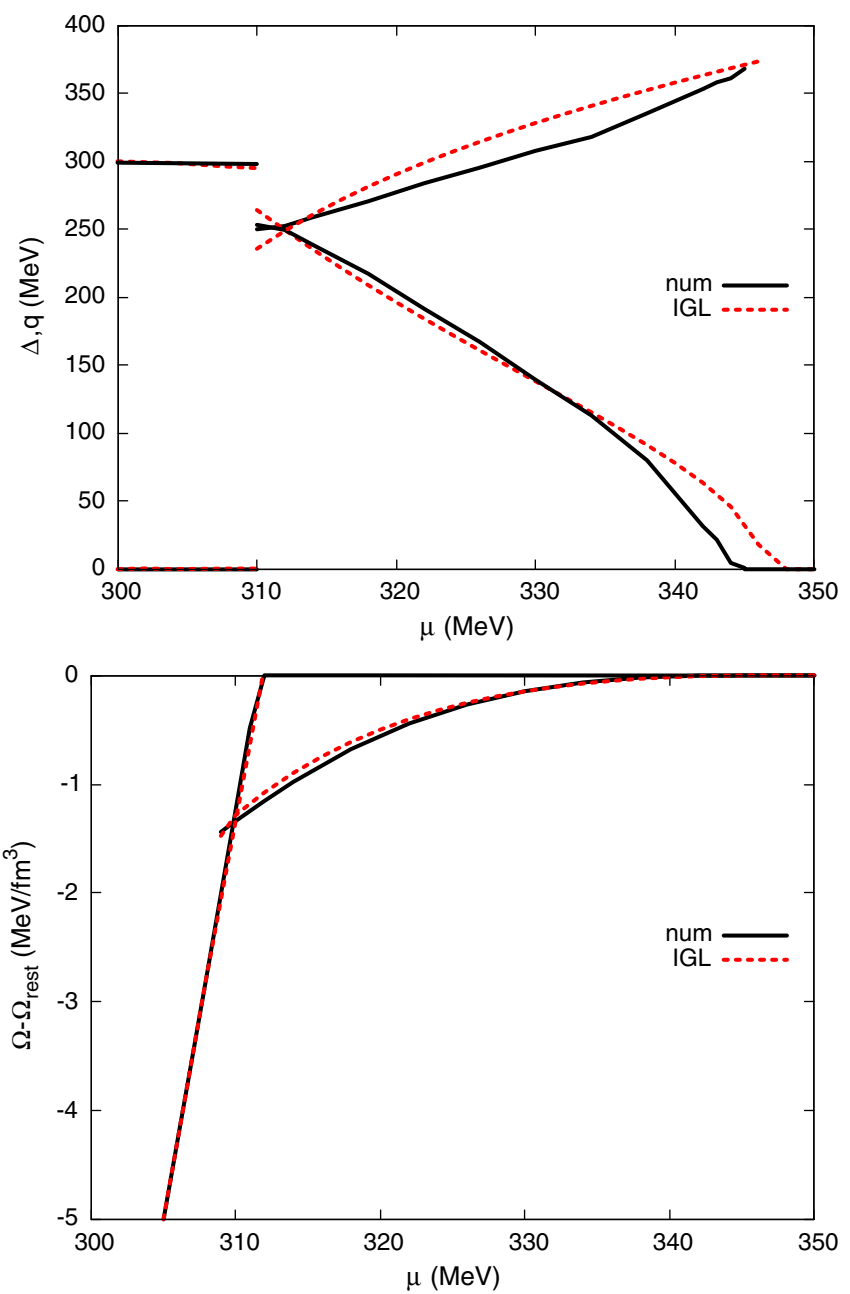

FIG. 5. Comparison of the IGL and numerical methods for the 2D cosine modulation ansatz, see Eq. (16), as a function of the quark chemical potential. Top: Values of the order parameters $\Delta$ and $q$. Bottom: comparison of the free-energy difference $\Omega-\Omega_{\text {rest }}$.

which can interpolate between a one-dimensional RKC modulation and a more involved two-dimensional structure. Consistent with our other results, we find that the minimum solution always corresponds to one of the two $\nu$ being zero, while the other reduces to the value obtained when minimizing with the one-dimensional ansatz Eq. (15).

Thus, as it was already found in Ref. [14], we can confirm within our novel approach that 2D modulations are disfavored with respect to 1D modulations at vanishing temperatures. We therefore expect that the same "hierarchy" found in Ref. [13] close to the Lifshitz point holds also at vanishing temperatures and that $3 \mathrm{D}$ modulations will thus be even further disfavored compared to twodimensional ones.

\section{QUALITATIVE ANALYSIS OF PAIRING}

The comparison between the considered 2D modulations and the 1D modulations suggests that the $1 \mathrm{D} \mathrm{RKC}$ is 


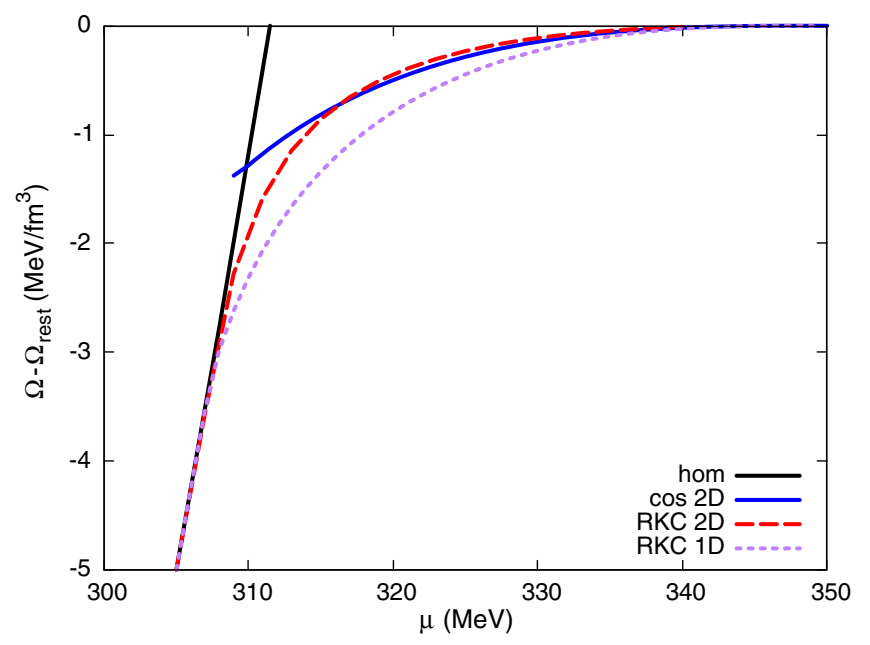

FIG. 6. Comparison of the free energies for a 1D RKC (15), a 2D cosine (16), and a 2D RKC (17), within the IGL approximation.

always favored. This result is in contrast with what is expected to occur in crystalline color superconductors, in which a crystalline 3D pattern seems to be favored [16,27]. It is believed that in color superconductors the occurrence of the crystalline phase is due to the maximization of pairing at the Fermi surface; indeed, the presence of a collective Fermi surface phenomenon seems to be the key point for obtaining a crystalline phase.

Quite generally, a certain modulation is energetically favored if the energy gain due to pairing is larger than the energy cost of having pairs with nonvanishing total momentum. Let us examine in detail what happens in an inhomogeneous $\chi \mathrm{SB}$ condensate. For a qualitative understanding of the phenomenon, we consider first the effect of a nonvanishing momentum, and then we allow for pairing. For understanding whether multidimensional pairing is favored, we consider what happens for a plane wave ansatz. As discussed in Refs. [6,16], one way of representing the Fermi surface effects is to inspect the integrand of the free energy, corresponding, in our case, to the integrand appearing in Eq. (7). In Fig. 7, we plot this function at $\mu=335 \mathrm{MeV}$, that is, within the inhomogeneous $\chi \mathrm{SB}$ window. The left panel corresponds to the free case, that is, $q=0$ and $\Delta=0$. The integrand is peaked at $p=\mu$, meaning that the larger contribution comes from the Fermi surface, corresponding to the lighter region in Fig. 7. This is the so-called pairing region, while the parts well inside the Fermi sphere or well outside it correspond to the blocking regions (see the discussion in Ref. [16] about pairing and blocking regions in color superconductors). In other words, pairing well inside/outside the Fermi sphere has a large free-energy cost, because particles should climb to the tip of the free energy (integrand), which is at the Fermi sphere. On the other hand, particle and hole excitations at the Fermi sphere are already at the tip of the mountain, that is, to the largest possible energy, and they can eventually pair at no cost to form a chiral condensate [28].

Now, we consider a momentum shift of the fermions. When pairs have nonvanishing total momentum, one can imagine first displacing fermions by $q$ and then to turn on pairing. This is exactly what one does when diagonalizing the full Hamiltonian for the single plane wave to obtain the free energy in Eq. (7). This procedure is discussed in detail in Ref. [16] for crystalline color superconductors, where it is shown how by a proper momentum shift the quark propagator becomes diagonal (see also Ref. [7] for an analogous discussion for inhomogeneous chiral condensates). This momentum shift has the effect of separating the Fermi spheres, as shown in the central panel of Fig. 7 for $q=241 \mathrm{MeV}$. Now, the only pairing region corresponds to the ribbon where the two Fermi spheres touch. This picture also explains why $q<\mu$; indeed, if this were not the case,
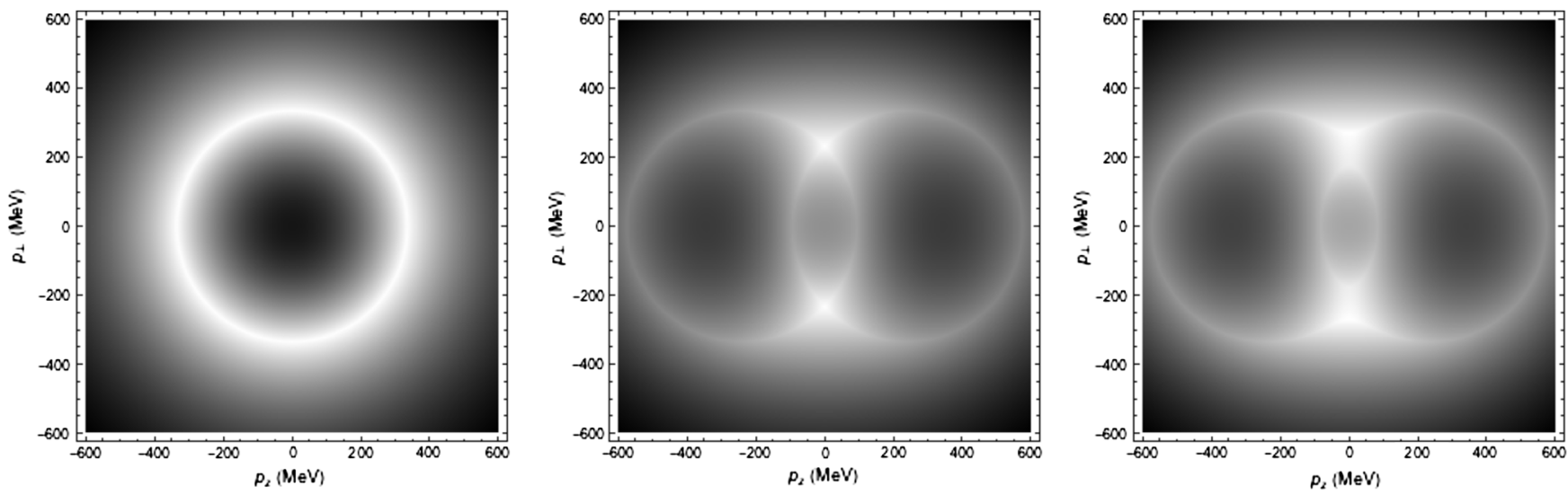

FIG. 7. Two dimensional contour plots of the integrand of the free energy for the CDW ansatz, Eq. (7). All the results are obtained for $\mu=335 \mathrm{MeV}$, but with different values of $q$ and $\Delta$. The lighter region corresponds to the region where the free energy cost for exciting quasiparticles is smaller. Left: unpaired phase, $q=0$ and $\Delta=0$. Center: $q=241 \mathrm{MeV}$ and $\Delta=0$. The effect of the large momentum $q$ is to strongly displace the Fermi spheres. Right: $q=241 \mathrm{MeV}$ and $\Delta=44 \mathrm{MeV}$ (corresponding to the energetically favored values at $\mu=335 \mathrm{MeV}$ ). The smearing of the lighter region is due to the pairing. 


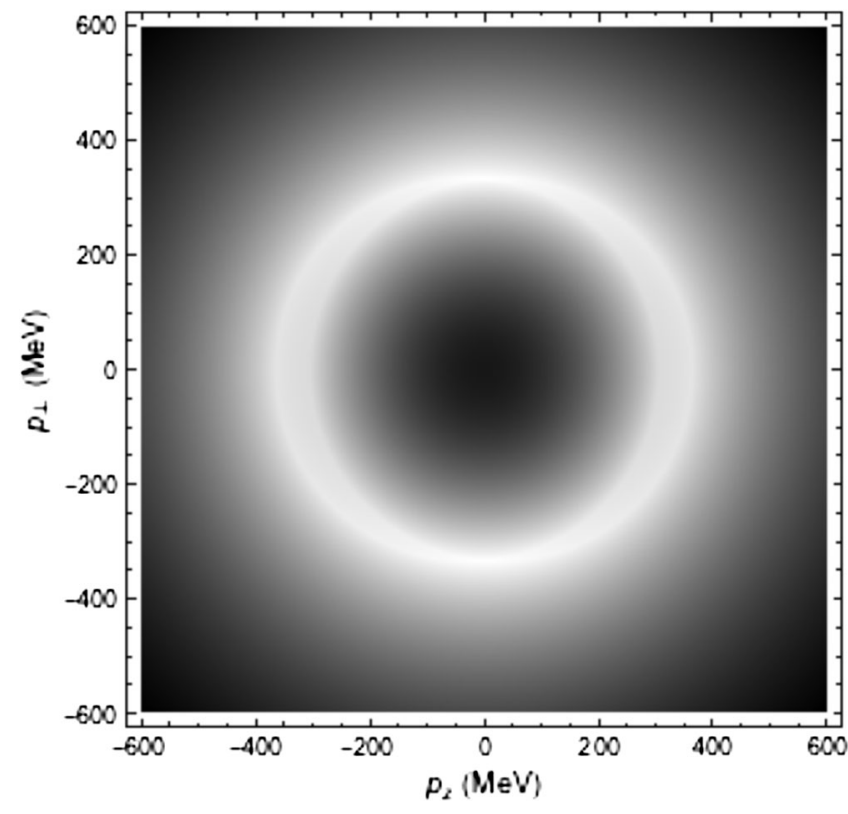

FIG. 8. Contour plots of the integrand of the free energy for CDW ansatz, Eq. (7). For $\mu=335 \mathrm{MeV}, q=30 \mathrm{MeV}$, and $\Delta=5 \mathrm{MeV}$.

the two Fermi spheres would have no overlapping regions. Exciting quasiparticles and/or holes in the pairing ribbon has no free energy cost, whereas particles from all other regions should climb an energy barrier. Indeed, we see that this is exactly what happens for $\Delta=44 \mathrm{MeV}$, right panel, where the smearing of the touching regions is exactly due to pairing. It is not possible now to add pairing in different regions of the Fermi spheres, say, in the region $p_{\perp} \sim 0$, because these regions are too far apart and therefore the free energy cost for exciting particles and/or holes would be too high. Therefore, multidimensional modulations are disfavored in the $\chi \mathrm{SB}$ phase because $q$ is too large.

This does not happen in color superconductors. Indeed, one important difference between the inhomogeneous $\chi \mathrm{SB}$ phase and the crystalline color superconductors regards the magnitude of $q$. Broadly speaking, $q$ has to be proportional to the stress exerted on the pairing mechanism. In the $\chi \mathrm{SB}$ phase, the stress is proportional to $\mu$, because pairing is related to the formation of a chiral condensate. On the other hand, in color superconductors, $q \propto \delta \mu$, where $\delta \mu \ll \mu$ is the mismatch between the Fermi spheres due to an imbalance between quarks of different flavors. For illustrative purposes, let us consider the non-energeticallyfavored $\chi \mathrm{SB}$ configuration corresponding to small $q$ and $\Delta$, somehow mimicking what happens in color superconductors. We show in Fig. 8 the integrand of Eq. (7), with $q=30 \mathrm{MeV}$ and $\Delta=5 \mathrm{MeV}$. Again, pairing can happen in the ribbon where the two Fermi spheres overlap. However, it is clear now that it would be possible to slightly modify the Fermi sphere for allowing pairing, say, in the $p_{\perp} \sim 0$ plane, at a small free-energy cost.

\section{CONCLUSIONS}

We have presented a novel approach to study spatially inhomogeneous pairing by an improved Ginzburg-Landau expansion, Eq. (2). This approach relies on a scale separation between long-wavelength fluctuations, dominating the transition to the homogeneous phase, and rapid fluctuations governing the transition to the chirally restored phase. The IGL reproduces correctly by construction the homogeneous limit and allows for a description of the chiral restoration transition from the inhomogeneous phase with arbitrarily high precision by a controlled gradient expansion.

We have applied the IGL to the study of the inhomogeneous $\chi \mathrm{SB}$ phase at $T=0$, reproducing the results obtained by numerical methods and extending the analysis to novel structures. These structures can hardly be studied by the numerical method, because of the complicated Fourier expansion technique underlying these methods. On the other hand, the IGL expansion turns out to be an extremely powerful tool, allowing us to quickly examine various crystalline structures to an arbitrarily accurate approximation. In this way, we checked that various $2 \mathrm{D}$ modulations are disfavored with respect to the 1D RKC one in Eq. (15), confirming and extending previous results obtained via brute-force numerical computations [14].

It is worth emphasizing that no approximate method so far has been used to analyze the $T=0$ case, probably because the standard GL approximation was assumed to be unreliable. Actually, we find that the GL approximation at $\mathcal{O}\left(\alpha_{8}\right)$ gives a surprisingly good qualitative agreement with the results of the full numerical computations. However, to make a quantitative comparison of the free energies of different structures, a refined approach must be used, and the IGL devised here performs this task excellently. In particular, we showed that it is able to give an accurate description of both the second-order phase transition to the chirally restored phase and of the phase transition to the homogeneous $\chi \mathrm{SB}$ phase. As it turns out, a small number of additional specific gradient terms is enough to provide excellent agreement with the numerical data.

Finally, it is worth recalling that fluctuations are expected to have a strong effect on the formation of inhomogeneous condensates [29-31], particularly in the case of lower-dimensional modulations [32] (see also Ref. [7] for a discussion). The inclusion of fluctuations in the IGL framework would lead to a systematic improvement beyond the mean-field approximation.

The present work can be extended in many different ways. The IGL free energy can be used to rapidly evaluate the free energy of various crystalline color superconducting configurations, as the ones considered in Ref. [10], and to extend the analysis to novel modulations. The only modifications needed in Eq. (2) are the replacement of the free 
energy of the homogeneous phase with the 2SC one (for two flavors) or of the color-flavor locked one (for three flavors) and to replace the $\alpha_{n}$ coefficients with the pertinent ones, which can, in principle, be obtained by considering modulations for which the eigenvalue spectrum is known, such as a simple Fulde-Ferrell-type plane wave $[6,33,34]$. In this case, one can also compare the IGL results with those obtained by the numerical method in Ref. [15]. We will shortly present results on this topic.
Moreover, the IGL can be modified to simultaneously include the chiral and diquark condensates for examining the coexistence of the inhomogeneous $\chi \mathrm{SB}$ and of the crystalline color superconducting phase. In this case, the color-superconducting phase is expected to arise where the chiral condensate is small or, equivalently, where the density is large. Since 1D chiral modulations are favored, we expect that a cosine modulation, see, for example, Ref. [6], could be favored.
[1] K. Fukushima and T. Hatsuda, Rep. Prog. Phys. 74, 014001 (2011).

[2] K. Rajagopal and F. Wilczek, At The Frontier of Particle Physics edited by M. Shifman and B. Ioffe (World Scientific, 2000), p. 2061-2151, DOI: 10.1142/ 9789812810458_0043.

[3] G. Aarts, Pramana 84, 787 (2015).

[4] S. P. Klevansky, Rev. Mod. Phys. 64, 649 (1992).

[5] M. Buballa, Phys. Rep. 407, 205 (2005).

[6] R. Anglani, R. Casalbuoni, M. Ciminale, N. Ippolito, R. Gatto, M. Mannarelli, and M. Ruggieri, Rev. Mod. Phys. 86, 509 (2014).

[7] M. Buballa and S. Carignano, Prog. Part. Nucl. Phys. 81, 39 (2015).

[8] D. Nickel, Phys. Rev. Lett. 103, 072301 (2009).

[9] M. G. Alford, K. Rajagopal, and F. Wilczek, Nucl. Phys. B537, 443 (1999).

[10] K. Rajagopal and R. Sharma, Phys. Rev. D 74, 094019 (2006).

[11] M. Thies, Phys. Rev. D 69, 067703 (2004).

[12] D. Nickel, Phys. Rev. D 80, 074025 (2009).

[13] H. Abuki, D. Ishibashi, and K. Suzuki, Phys. Rev. D 85, 074002 (2012).

[14] S. Carignano and M. Buballa, Phys. Rev. D 86, 074018 (2012).

[15] D. Nickel and M. Buballa, Phys. Rev. D 79, 054009 (2009).

[16] M. Mannarelli, K. Rajagopal, and R. Sharma, Phys. Rev. D 73, 114012 (2006).

[17] Y. Nambu and G. Jona-Lasinio, Phys. Rev. 122, 345 (1961).

[18] F. Dautry and E. Nyman, Nucl. Phys. A319, 323 (1979).
[19] E. Nakano and T. Tatsumi, Phys. Rev. D 71, 114006 (2005).

[20] M. Buballa and S. Carignano, Phys. Rev. D 87, 054004 (2013).

[21] S. Carignano and M. Buballa, Acta Phys. Pol. B Proc. Suppl. 5, 641 (2012).

[22] S. Carignano, M. Buballa, and B.-J. Schaefer, Phys. Rev. D 90, 014033 (2014).

[23] S. Carignano, D. Nickel, and M. Buballa, Phys. Rev. D 82, 054009 (2010).

[24] M. Buballa and S. Carignano, Eur. Phys. J. A 52, 57 (2016).

[25] D. G. Ravenhall, C. J. Pethick, and J. R. Wilson, Phys. Rev. Lett. 50, 2066 (1983).

[26] T. Kojo, Y. Hidaka, K. Fukushima, L. D. McLerran, and R. D. Pisarski, Nucl. Phys. A875, 94 (2012).

[27] M. G. Alford, J. A. Bowers, and K. Rajagopal, Phys. Rev. D 63, 074016 (2001).

[28] T. Kojo, Y. Hidaka, L. McLerran, and R. Pisarski, Nucl. Phys. A843, 37 (2010).

[29] T.-G. Lee, E. Nakano, Y. Tsue, T. Tatsumi, and B. Friman, Phys. Rev. D 92, 034024 (2015).

[30] R. Yoshiike, T.-G. Lee, and T. Tatsumi, Phys. Rev. D 95, 074010 (2017).

[31] Y. Hidaka, K. Kamikado, T. Kanazawa, and T. Noumi, Phys. Rev. D 92, 034003 (2015).

[32] L. D. Landau and E. M. Lifshitz, Statistical Physics Part 1, Course of Theoretical Physics (Pergamon, New York, 1969).

[33] P. Fulde and R. A. Ferrell, Phys. Rev. 135, A550 (1964).

[34] A. I. Larkin and Y. N. Ovchinnikov, Zh. Eksp. Teor. Fiz. 47, 1136 (1964) [Sov. Phys. JETP 20, 762 (1965)]. 